\title{
Review of: "On limits of contact tracing in epidemic control"
}

\author{
Leonardo López
}

Potential competing interests: The author(s) declared that no potential competing interests exist.

The article presents good work about the impact of contact tracing and quarantine in the evolution of the epidemic. To do so, the authors proposed a deterministic model that defines a simple rule on the reproduction number $\mathrm{R} 0$ in terms of ratio of diagnosed cases and, quarantine and transmission parameters. The proposed model is applied to the early stage of the Covid-19 crisis in Poland. They investigate 3 scenarios corresponding to different ratios of diagnosed cases. Their results show that, depending on the scenario, contact tracing prevented from $50 \%$ to over $90 \%$ of cases. The effects of quarantine are limited by the fraction of undiagnosed cases. The key conclusion is that under realistic assumptions the epidemic can not be controlled without any social distancing measures.

Two issues of this work do not convince me. The first of these is the message itself: I do not know if it is the objective of the work or not but it gives the feeling when one is reading the article that the objective is to raise the uselessness of sanitary restrictions, blockades, quarantines, and others. From what I see they place too much importance on tracking active cases and suggested that they could relax or remove other measures by increasing these traces. This seems to me to be a somewhat dangerous message, especially because I can't see that this system is $100 \%$ reliable and I don't know if it is a system that can be applied everywhere. On the other hand, several studies confirm that mixed strategies where mobility between populations is limited are more efficient and in any case would also be strongly conditioned by the social characteristics of the population where they are applied.

As the first example of this can be mentioned in the work of Liu et al.

(https://doi.org/10.1016/j.rinp.2021.104305) in which the authors introduce a mathematical model to evaluate and compare the effects and intensity of the coronavirus disease 2019 prevention and control measures in different Chinese provinces. This work concludes that comprehensive intervention measures, in which social distancing measures may have played a greater role than isolation measures, resulted in a reduction of new cases. In this context, Shanghai had the highest intervention intensity. In the context of the global coronavirus disease, the authors conclude prevention and control experience of some key areas in China (such as Shanghai and Guangdong) provide references for outbreak control in many countries. Between those measures, lock-down and quarantine are the more important. 
Another work that follows the same line is the one performed by Leung et al.

(https://doi.org/10.1016/S0140-6736(20)30746-7). In this work the authors estimated the instantaneous reproduction number $(R \mathrm{t})$ of COVID-19 in Beijing, Shanghai, Shenzhen, Wenzhou, and the ten Chinese provinces that had the highest number of confirmed COVID-19 cases; and the confirmed case-fatality risk (CFR) in Beijing, Shanghai, Shenzhen, and Wenzhou, and all 31 Chinese provinces. They use a SIR-like model to show the potential effects of relaxing containment measures after the first wave of infection. In all selected cities and provinces, the $R$ t decreased substantially since Jan 23 , when control measures were implemented, and have since remained below 1 . The CFR outside Hubei was $0.98 \%(95 \% \mathrm{Cl} 0.82-1 \cdot 16)$, which was almost five times lower than that in Hubei (5.91\%, 5.73-6.09). Relaxing the interventions (resulting in $R t>1$ ) when the epidemic size was still small would increase the cumulative case count exponentially as a function of relaxation duration, even if aggressive interventions could subsequently push disease prevalence back to the baseline level. This work found that the first wave of COVID-19 outside of Hubei has abated because of aggressive non-pharmaceutical interventions.

In another work performed by Ye et al. (http://dx.doi.org/10.2471/BLT.20.251561) the authors take the epidemic trends (daily incidence rate of confirmed coronavirus cases) of 51 cities between January 18 and February 19 of 2020, They also took into account the degree of the control measures among 51 cities by a three-dimensional score constructed according to a panel of 12 indicators, including socioeconomic status, population flows and implemented measures (i.e., emergency preparedness, public facility restrictions, travel restrictions, and community engagement). In this study, they found four patterns of epidemic trends: High incidence maintaining, low starting and then recurring, moderately high incidence, and moderately low incidence. The scores for these control measures were different among the 4 clusters and across cities within the same cluster. They found by doing this analysis the COVID-19 epidemic trends were different among Chinese cities and those trends could be affected by socioeconomic status, population flows, and implemented measures with a high influence on population flows within and between cities.

Finally, the work by Lin et al. authors develop a model to explain in a theoretical way the impact mechanism of prevention and control measures on the spread of the COVID-19 epidemic. They divided the measures taken in China into two categories: on one hand the mobility restrictions and, on the other hand, non-pharmacological interventions (O-NPI). They found that although both of the two measures play a good role in controlling the development of the epidemic, the effect shows a significant difference in different regions, and both the two measures had no significant effects in low-risk regions. To go further, they prove that measures taken in a low-risk region are mainly against the imported cases, while a highrisk region has to defend against both imported cases and spread from within, so tracing and isolation of cases has a great effect on the control; The rapid and accurate transmission of information, a higher protection awareness of the public, and stronger confidence of residents can promote the implementation of the measures proposed here. 
Taking into account the aforementioned works, from the point of view of the hypotheses raised in the analyzed work and the findings made by other authors with a much more heterogeneous diversity of data than those used for the study in Poland, it can be said that putting everything the effort in a single control strategy is not efficient to control a pandemic of such rapid dynamics as the one we have witnessed.

Finally, and strictly from the point of view of the model used, it should be noted that the authors do not model deaths and the impact that control measures may have on mortality from the virus.

On the other hand, it is not seen that there is a sensitivity analysis of the parameters of the model (deterministic), thus not being able to determine the real impact that these have on the output of the model.

The fit of the model is superficially explained and they do not give too many specifications about the configuration of the algorithm used, they only say that it is a gradient method and that the fitted function is the mean square error of the reported cases. They do not explain how they initialize it, step size, optimization algorithm, integration, among other things 